\title{
Performance Analysis of an Ultra Dense Network with and without Cell Cooperation
}

\author{
Aijun Cao, Yonghong Gao, Pei Xiao, Rahim Tafazolli \\ \{cao.aijun,gao.yonghong\}@zte.com.cn, \{p.xiao,r.tafazolli\}@surrey.ac.uk \\ ZTE Wistron Telecom AB \\ 19F, Farogatan 33, Kista Science Tower \\ Institute for Communication Systems (ICS) \\ University of Surrey \\ 16451 Kista, Sweden, \\ Guildford, Surrey GU2 7XH, UK
}

\begin{abstract}
This paper presents an analysis on performance of an ultra dense network (UDN) with and without cell cooperation from the perspective of network information theory. We propose a UDN performance metric called Total Average Geometry Throughput which is independent from the user distribution or scheduler etc. This performance metric is analyzed in detail for UDN with and without cooperation. The numerical results from the analysis show that under the studied system model, the total average geometry throughput reaches its maximum when the inter-cell distance is around $6 \sim 8$ meters, both without and with cell cooperation. Cell cooperation can significantly reduce intercell interference but not remove it completely. With cell cooperation and an optimum number of the cooperating cells the maximum performance gain can be achieved. Furthermore, the results also imply that there is an optimum aggregate transmission power if considering the energy cost per bit.
\end{abstract}

\section{INTRODUCTION}

As mobile end users spend more and more time everyday in applications installed in smart handsets, such as social networking, mobile payments and entertainment etc., the demand for higher data rates and volumes for mobile broadband is constantly increasing. As one of the pioneer projects on 5G, METIS proposes the following general targets for user rates and volumes per area respectively [1]:

- 10 times to 100 times higher typical user data rate

- 1000 times higher mobile data volume per area

The spectrum efficiency provided by a single radio link has been significantly increased from $3 \mathrm{G}$ to $4 \mathrm{G}$ by exploration of different techniques, and approaching its theoretical limit. Thus the increase of throughput for a single radio link relies on the acquisition of more spectrums, and on the reduction of signal energy loss due to e.g., path-loss and aggressive frequency reuse. The latter can be achieved by cell densification, which means deploying many more nodes in a given area. Moreover, spatial reuse can be increased with cell densification. Apparently it is one of the most promising ways to improve the mobile data volume per area, which justifies the topic of ultra dense network (UDN) being one of the horizontal topics in METIS [2], [8].

There are many challenges as the sites become more closely deployed. One issue is that inter-cell interference becomes much more severe compared with the case with a larger cell radius, and it might completely offset the gain from the cell densification if not handled appropriately. Cooperation among cells can effectively improve the situation.

For a wireless network deployed in a specific area, the achievable throughput in the area (labelled as aggregate throughput) depends on many parameters, such as the number of sites, the transmit power of each cell, the number of users and the distribution of the users, the user traffic, the geographical environments, the scheduler, and the cooperative operations among the sites, etc. A theoretical analysis of the aggregate throughput becomes increasingly complicated when more network parameters that are taken into account. Several previous analytical studies on UDN performance focus on the performance in terms of data rate of either a single radio link, or single cell spectrum efficiency [3], [6], [7], [9], [10]. Other analytical works portray the network spectrum efficiency [4], [5], but the results are dependent on either a specific user distribution, or a specific scheduler.

In this paper, we conduct an analysis on the performance of a UDN both with and without cell cooperation, from an information theoretical perspective. The performance gain and the limits of cell densification and cooperation are studied.

The remainder of the paper is organized as follows. Sec. II describes the system model. The performance analysis in the case without cell cooperation is presented in Sec. III, and in the case with cell cooperation in Sec. IV. The numerical results are shown in Sec. V. Finally, conclusions are drawn in Sec. VI.

\section{SYSTEM MODEL}

In this paper, we focus on the network performance considering the total data volume provided by a UDN consisting of many cells, aiming to obtain a theoretical analysis solely from information theory perspective on the UDN network performance independent from the user distribution and the scheduler etc. In order to facilitate the analysis, we make the following assumptions:

(1) Regularized site deployment:

- a square zone with $D$ meters length in both the $\mathrm{x}$ and $\mathrm{y}$ directions. Note that the assumed square zone instead of the usual hexagonal cell structure is mainly for the convenience of computing the throughput per unit zone, but it is not a prerequisite for the analysis. 
- equal distance between adjacent sites in the $\mathrm{x}$ direction as well as in the $y$ direction,

- $\quad$ the total number of sites, $N=Q^{2}$, where $Q$ is the number of sites in the $\mathrm{x}$ direction as well as in the y direction.

- Equal downlink transmit power for each cell

(2) Downlink is transmitted with full power from each cell. The transmission is isotropic with a flat power density in the frequency domain

(3) The same path-loss model, and the same minimum distance between a cell and a UE (User Equipment) $D_{\text {min }}$, is applied to all of the cells

Fig. 1 shows one example of the regularized UDN deployment described above.

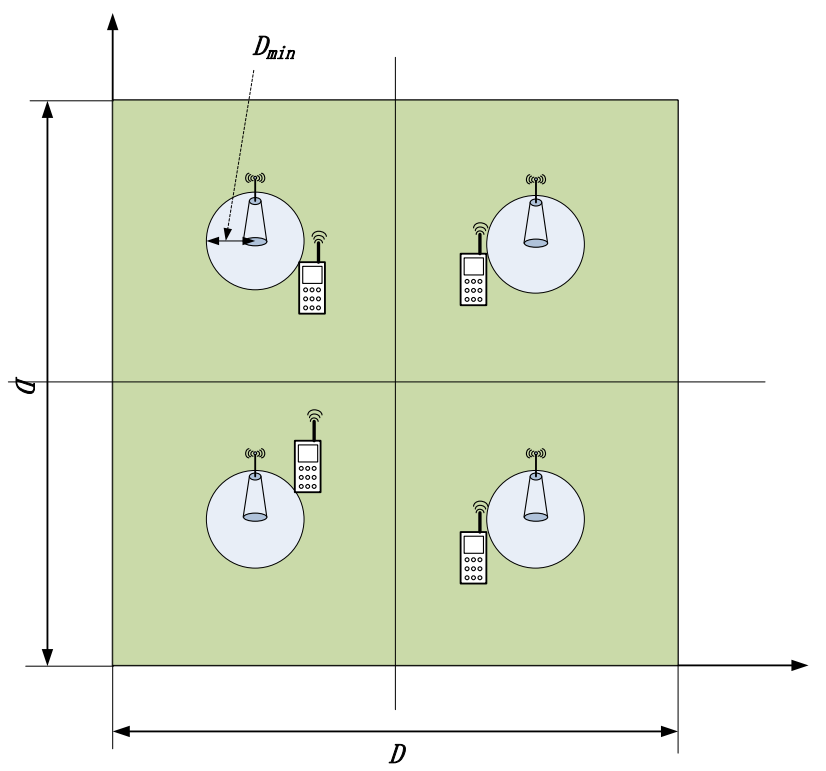

Figure 1 Regularized UDN deployment

\section{ANALYSIS OF UDN PERFORMANCE WITHOUT COOPERATION}

We propose a performance measurement called "average geometry throughput". It can be computed according to the following method, which is independent from the choice of scheduler, user number and distribution etc. Note that the measurement can be readily extended to non-regularized UDN deployment as well.

- Assume that a considered point $(x, y)$ is located in the cell $c$, which means that the power received from cell $c$ is considered as useful signal power, whereas the received power from all other cells is regarded as interference

- The received SINR of the point is $\rho(\mathrm{x}, \mathrm{y})=\frac{\mathrm{P} / \mathrm{PL}_{\mathrm{c}}}{\sum_{\mathrm{i} \neq \mathrm{c}}^{\mathrm{N}} \frac{\mathrm{P}}{\mathrm{PL}_{\mathrm{i}}}+\sigma^{2}}$, where $P$ is the transmission power, $P L_{c}$ is the path-loss from cell $c$ to the point, and $\mathrm{PL}_{\mathrm{i}}$ is the path-loss from all other interfering cells to the point, $N$ is the total number of cells, and $\sigma^{2}$ is the background noise power

- The geometry throughput corresponding to the point ( $x$, $y$ ) is calculated according to the Shannon equation as:

$$
\begin{aligned}
& G_{C}(x, y)=B \cdot \log _{2}(1+\rho(x, y)) \\
& =B \cdot \log _{2}\left(1+\frac{P / \mathrm{PL}_{c}}{\sum_{i \neq C}^{N} \frac{P}{P_{i}}+\sigma^{2}}\right)
\end{aligned}
$$

where $B$ is the bandwidth of the transmission.

- The average geometry throughput of the cell $c$ as shown in Eq. (2):

$$
A(c)=\frac{1}{R} \oiint_{\text {Cell c }} G_{c}(x, y) d x d y
$$

where $R$ is the total effective area of the cell $c$.

Then it is natural to calculate the Total Average Geometry Throughput in one area by summing the average geometry throughput of all the cells in the area as seen in Eq. (3):

$$
\mathrm{T}(\mathrm{N})=\sum_{\mathrm{c}=1}^{\mathrm{N}} \mathrm{A}(\mathrm{c})
$$

As the number of deployed cells $N$ in an area increases, the total average geometry throughput starts to rise, gradually reaches its maximum and then starts to decrease due to more severe interference. The numerical results will be presented in Sec. V.

\section{ANALYSIS OF UDN PERFORMANCE WITH COOPERATION}

Similarly to the case without cooperation, we can further extend the geometry throughput at the point $(x, y)$ provided that $M$ closest cells $\left\{c_{1}, c_{2}, \ldots, c_{M}\right\}$ are jointly transmitting to the point (Note that $M$ is also labelled as "CoMP level" in this paper)

The received SINR can be expressed as Eq. (4):

$$
\rho(x, y)=\frac{\left.\sum_{i=\left\{c_{1}, \ldots, c_{M}\right\}}\right\} \frac{P_{i}}{\mathrm{PL}_{i}}}{\sum_{i \neq\left\{c_{1}, \ldots, c_{M}\right.}^{N} \frac{P}{\mathrm{PL}_{i}}+\sigma^{2}}
$$

where $P_{i}$ is the transmission power of cell $c_{i}$ intended for the point in the cooperative operation, and $\sum_{\mathrm{i}=\left\{\mathrm{c}_{1}, \ldots, \mathrm{c}_{\mathrm{M}}\right\}} \mathrm{P}_{\mathrm{i}}=\mathrm{P}$, which means that the total transmit power to the point does not change with the cell cooperation, compared with the case without cell cooperation.

Then the geometry throughput for this point with cooperative operation can be rewritten as Eq. (5):

$$
\begin{aligned}
& \mathrm{G}_{\mathrm{C}}(\mathrm{x}, \mathrm{y})=\mathrm{B} \cdot \log _{2}(1+\rho(\mathrm{x}, \mathrm{y})) \\
& =\mathrm{B} \cdot \log _{2}\left(1+\frac{\sum_{\mathrm{i}=\left\{\mathrm{c}_{1}, \ldots, \mathrm{c}_{\mathrm{M}}\right\} \frac{\mathrm{P}_{\mathrm{i}}}{\mathrm{PL}_{\mathrm{i}}}}}{\left.\sum_{\mathrm{i} \neq\left\{\mathrm{c}_{1}, \ldots, \mathrm{c}_{\mathrm{M}}\right\}}\right\} \frac{\mathrm{P}}{\mathrm{PL}_{\mathrm{i}}}+\sigma^{2}}\right)
\end{aligned}
$$

The average geometry throughput of the cell $\mathrm{c}$ is computed as Eq. (2) and finally the total average geometry throughput is obtained according to Eq. (3).

With the cell cooperation, on one hand, it can enhance the receive signal power by turning the inter-cell interference into useful signal thus contributing to increased total throughput in the area. On the other hand, it can have a negative impact on the total throughput in the area due to the fact that the cell cooperation consumes more spectrums and power in the cells involved in the cooperation, thus reduces the overall frequency reuse. This implies that there exists an optimum number of cells in the cooperation. 


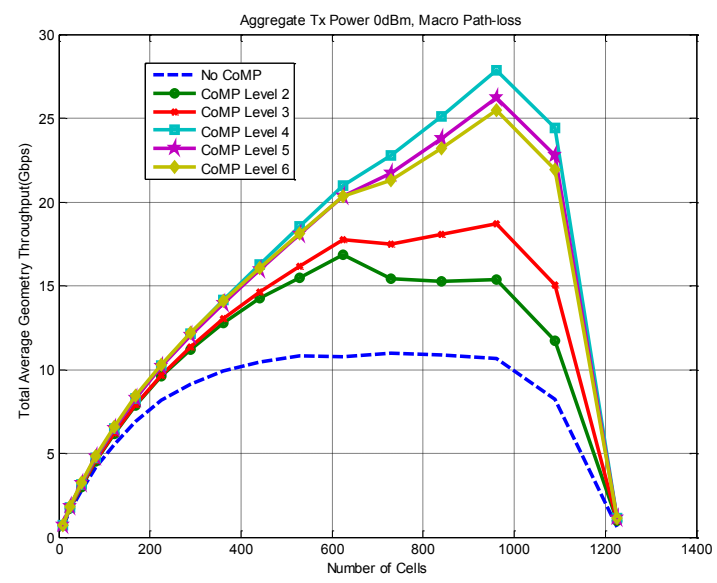

(a) Macro path-loss model

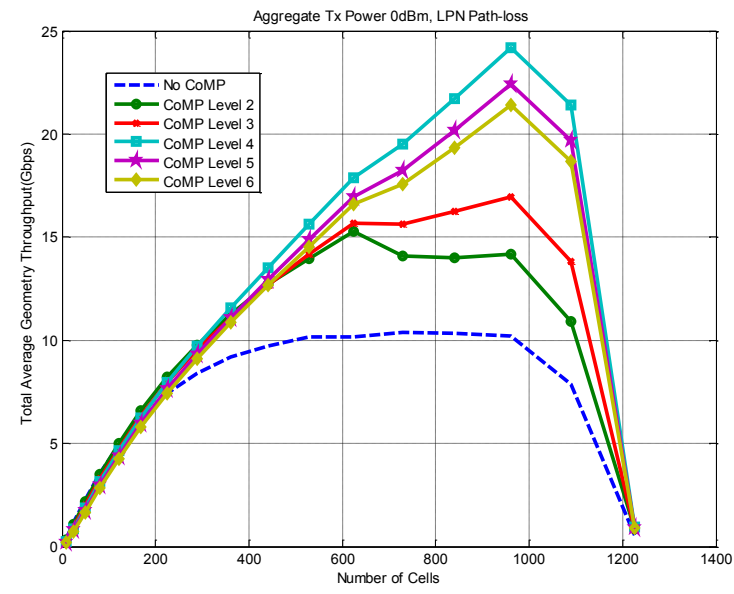

(b) LPN path-loss model

Figure 2 Cell densification gain

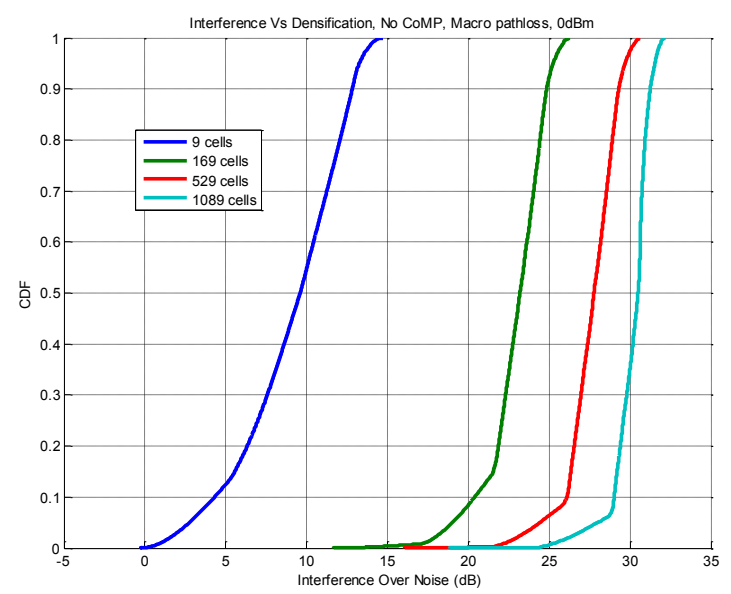

(a) Cumulative distribution function for the case without cell cooperation

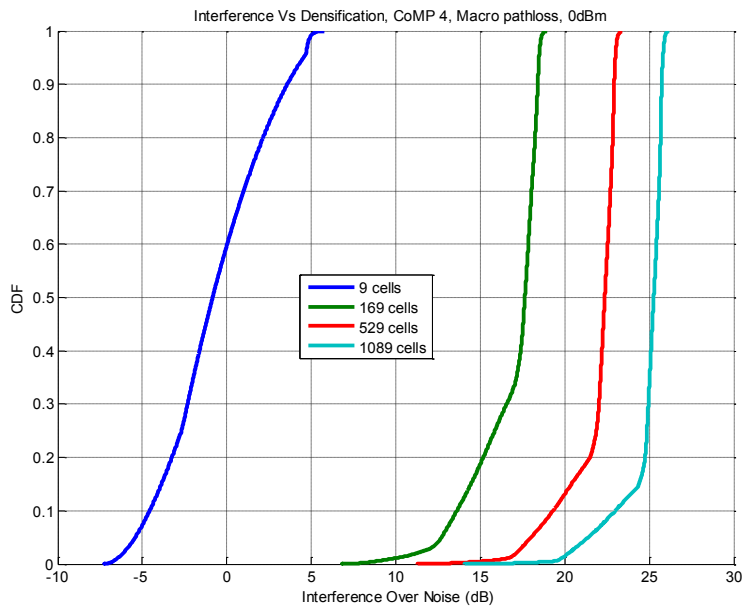

(b) Cumulative distribution function for the case with CoMP level $=4$

Figure 3 Interference Vs Densification

\section{NUMERICAL RESULTS}

Under the parameter setup shown in Table 1, we perform numerical integration of the above equations (note that this is not a system simulation). It is also worth pointing out that total transmission power of the concerned area (the aggregate transmission power) is kept constant. This means that the transmission power of each individual cell is reduced when more nodes are deployed into the area. The achieved gain from the cell densification can be shown.

Table 1. Parameters for numerical results

\begin{tabular}{|c|c|}
\hline Parameter & Value \\
\hline Area size & $200 \times 200$ meters \\
\hline Number of cells, $N$ & $\{9,25,49, \ldots, 1089,1225\}$ \\
\hline $\begin{array}{c}\text { Aggregate } \\
\text { transmission power } \\
\text { of the area }\end{array}$ & $\{-20,-15,-10,0,10\} \mathrm{dBm}$ \\
\hline Noise figure & $5 \mathrm{~dB}$ \\
\hline$D_{\min }$ & 4 meters \\
\hline
\end{tabular}

\begin{tabular}{|c|c|}
\hline Bandwidth, $B$ & $20 \mathrm{MHz}$ \\
\hline Carrier & $2 \mathrm{GHz}$ \\
\hline Noise power density & $-174 \mathrm{dBm} / \mathrm{Hz}$ \\
\hline & $\mathrm{PL}(\mathrm{dB})=128.1+37.6 \lg (\mathrm{R})$, \\
& $\mathrm{R}$ in km for Macro pathloss \\
Path-loss model [11] $\mathrm{PL}(\mathrm{dB})=140.7+36.7 \lg (\mathrm{R})$, \\
& $\mathrm{R}$ in km for LPN pathloss \\
\hline Cooperative $^{1}$ & $\begin{array}{c}\text { Equal power (non-coherent) } \\
\text { operation }\end{array}{ }^{1}$ \\
\hline
\end{tabular}

Fig. 2 shows how the total average geometry throughput varies with the degree of cell density with different cell cooperation levels and macro and LPN path-loss models. The figure shows that the gain from the cell densification reaches its peak value when there are $600 \sim 900$ cells (e.g., inter-site

\footnotetext{
${ }^{1}$ Results for other types of cooperative operation, e.g., selective node transmission, path-loss reverse proportional transmission etc., are not shown in this paper.
} 
distance is around $6 \sim 8$ meters) for both without and with cell cooperation. The maximum gain from the cell cooperation for both path-loss models is around $150 \%$, e.g., $2.5=$ $(27.8378 / 10.9858)$ for Macro case, and $2.3=$ (24.1846/10.3852) for LPN case.

Fig. 3 illustrates the cumulative distribution (CDF) curves of interference over the thermal noise for both noncooperative and cooperative operation under macro path-loss model. It can be observed that the cell cooperation can significantly improve the situation of the inter-cell interference, e.g., more than $5 \mathrm{~dB}$ in the figure. Fig. 2, however, indicates that the cell cooperation cannot completely resolve the inter-cell interference issue.

Techniques such as massive antenna/beam-forming could be used to further reduce the inter-cell interference and improve the average geometry throughput. This will be a research topic in our future works.

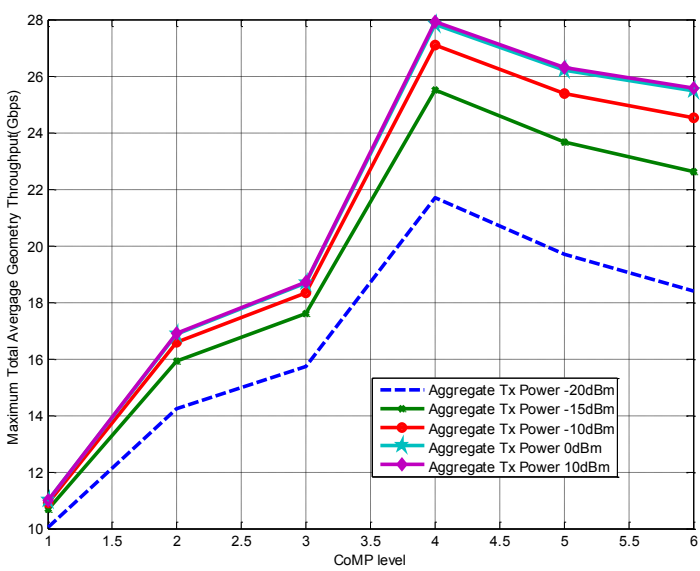

Figure 4 Optimum CoMP level

Fig. 4 justifies the statement on the optimum number of cooperative cells in Sec. IV and indicates that for different aggregate transmission power levels the optimum number is 4 in the studied system model.

Fig. 5 shows that the maximum total average geometry throughput quickly saturates with the increase of the aggregate transmission power, therefore an optimum aggregate transmission power value can be selected if considering the energy cost per bit.

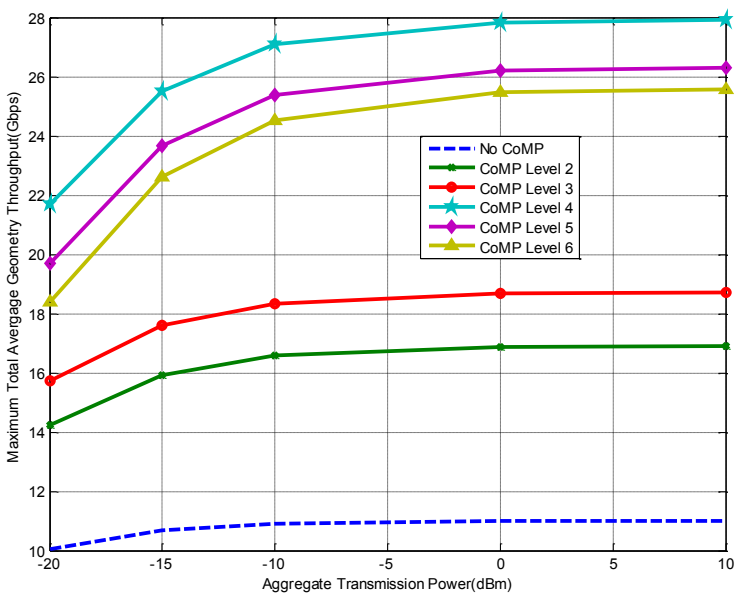

Figure 5 Power efficiency

\section{CONCLUSIONS}

This paper proposes a concept called total average geometry throughput as the performance index of the UDN, which is independent from any user distribution or scheduler etc. Detailed analysis based on this concept on the studied system model is made for both with and without joint transmission. The numerical results from the analysis show the total average geometry throughput reaches its peak value when the inter-site distance is around $6 \sim 8$ meters, even for the case with cell cooperation. It is also observed that the cell cooperation can significantly improve inter-cell interference but not completely resolve the situation. The best achievable throughput gain of $150 \%$ can be anticipated with an optimum number of 4 cooperating cells. Furthermore, the results also imply that the maximum total average geometry throughput quickly saturates with the increase of the aggregate transmission power, thus there is an optimum aggregate transmission power if considering the energy costs per bit.

\section{ACKNOWLEDGEMENTS}

The authors thank to Thorsten Schier, Jan Johansson and Patrick Svedman of ZTE Wistron Telecom AB for their intensive and valuable comments and fruitful discussions.

\section{REFERENCES}

[1] ICT-317669-METIS/D1.1, Scenarios, requirements and KPIs for 5G mobile and wireless system, METIS, Apr 30, 2013

[2] ICT-317669-METIS/D6.2, Initial report on horizontal topics, first results and 5G system concept, METIS, Mar 31, 2014

[3] A. Lozano, R. W. Heath, Jr., Jeffrey G. Andrews, "Fundamental limits of Cooperation", IEEE Trans. on Information Theory, vol 59, no. 9, Sep 2013, pp $5213-5226$

[4] Koudouridis, G.P., "On the capacity and energy efficiency of network scheduling in future ultra-dense networks," Computers and Communication (ISCC), 2014 IEEE Symposium on , vol. Workshops, no., pp.1,6, 23-26 June 2014

[5] Li, Qian Clara; Wu, Geng; Hu, Rose Qingyang, "Analytical study on network spectrum efficiency of ultra dense networks," Personal Indoor and Mobile Radio Communications (PIMRC), 2013 IEEE 24th International Symposium on, vol., no., pp.2764,2768, 8-11 Sept. 2013 
[6] Naumov, V.; Samouylov, K.; Sopin, E.; Andreev, S., "Two approaches to analyzing dynamic cellular networks with limited resources," Ultra Modern Telecommunications and Control Systems and Workshops (ICUMT), 2014 6th International Congress on , vol., no., pp.485,488, 6-8 Oct. 2014

[7] Stefanatos, S.; Alexiou, A., "Access Point Density and Bandwidth Partitioning in Ultra Dense Wireless Networks," Communications, IEEE Transactions on , vol.62, no.9, pp.3376,3384, Sept. 2014

[8] Baldemair, R.; Irnich, T.; Balachandran, K.; Dahlman, E.; Mildh, G.; Sel??n, Y.; Parkvall, S.; Meyer, M.; Osseiran, A., "Ultra-dense networks in millimeter-wave frequencies," Communications Magazine, IEEE, vol.53, no.1, pp.202,208, January 2015

[9] Bai, Lu; Liu, Tingting; Chen, Zhilin; Yang, Chenyang, "A graph-based interference topology control for ultra-dense networks," Signal Processing (ICSP), 2014 12th International Conference on , vol., no., pp.1676,1681, 19-23 Oct. 2014

[10] Sarret, M.G.; Catania, D.; Frederiksen, F.; Cattoni, A.F.; Berardinelli, G.; Mogensen, P., "Improving link robustness in 5G ultra-dense small cells by hybrid ARQ," Wireless Communications Systems (ISWCS), 2014 11th International Symposium on , vol., no., pp.491,495, 26-29 Aug. 2014

[11] 3GPP TR36.814 v9.0.0, "Further advancements for E-UTRA physical layer aspects", Mar 2010 